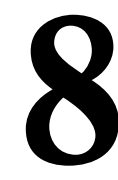

\title{
APORTES TEÓRICOS DE LA PERSPECTIVA DE GÉNERO EN LA MEJORA DE LA EDUCACIÓN DE LAS NIÑAS EN ÁFRICA
}

\section{(THEORETICAL CONTRIBUTIONS OF THE GENDER APPROACH TO IMPROVE EDUCATION FOR AFRICAN GIRLS)}

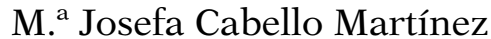

Irene Martínez Martín

Universidad Complutense de Madrid

DOI: $10.5944 / e d u c X X 1.17507$

\section{Cómo referenciar este artículo/How to reference this article:}

Cabello Martínez, M.J. y Martínez Martín, I. (2017). Aportes teóricos de la perspectiva de género en la mejora de la educación de las niñas en África. Educación XX1, 20(1), 163-181, doi: 10.5944/ educXX1.17507

Cabello Martínez, M.J. \& Martínez Martín, I. (2017). Aportes teóricos de la perspectiva de género en la mejora de la educación de las niñas en África. [Theoretical contributions of the gender approach to improve education for African girls]. Educación XX1, 20(1), 163-181, doi: 10.5944/ educXX1.17507

\section{RESUMEN}

Este artículo es parte de una investigación que pretende encontrar respuestas al interrogante: ¿qué aporta la inclusión de una perspectiva de género postcolonial y africana a la mejora del desarrollo y la educación de las niñas, en la construcción de sociedades más justas e igualitarias? La finalidad principal del mismo es la fundamentación y la argumentación respecto a la conexión entre los beneficios aportados por la perspectiva de género y una determinada forma de hacer educación.

El método de investigación seguido se basa en los principios cualitativos de estudio, el análisis documental, bibliográfico y de experiencias para definir el estado de la cuestión, los informes de investigaciones anteriores y las prácticas reflexivas para la acción; todo ello orientado a descifrar y redefinir el valor añadido que otorga un enfoque de género postcolonial y transversal, basado en investigaciones africanistas, para una acción educativa transformadora y 
crítica.

Como principales resultados y conclusiones establecemos que la educación no permanece al margen de los debates en torno a la construcción de sociedades más equitativas en favor de la justicia social, sino que se inserta en prácticas promovidas por enfoques sociales alternativos que pretenden la descolonización de los saberes respecto de las lógicas dominantes. Entre las contribuciones de estos nuevos paradigmas sociales encontramos las aportaciones que los feminismos postcoloniales y africanos hacen a la ciudadanía, destacando las relacionadas con perspectiva de género, desarrollo, empoderamiento y educación, tales aportaciones nos permiten avanzar en la definición de una forma particular de saber, ser y hacer que oriente la acción educativa.

\section{PALABRAS CLAVE}

Educación; equidad; género; desarrollo; África.

\section{ABSTRACT}

The purpose of this paper try to answer the question: what is the best part of the inclusion of a postcolonial African gender approach for improving female development and education for more equal and just societies? The main objective of this paper is the connection between the benefits provided by the gender perspective and a specific way to educate. Therefore, we'll try to connect the benefits of a gender and postcolonial approach, based on African studies, with the education for a social and equal development for girls and boys.

The research method followed for achieving these goals is based on the study of qualitative principles, documentary analysis, research reports and reflective practices for action; the postcolonial and gender approach is based on African research which attempts to transform the educational activity and the equality in development. The main results and conclusions of the paper establish that education is the center of the discussions about the need for an equitable society with social justice. Regarding that, we need different social approaches from dominant thinking. Among the contributions of these new social paradigms, we find the contributions that African and postcolonial feminism make to citizenship. This approach puts emphasis on topics such as gender, development, empowerment and education. These different topics will define a particular way to know, to be and to act in favor of a social justice.

\section{KEYWORDS}

Basic education; equality; gender; development; Africa. 
«No hay barrera, cerradura, ni cerrojo que puedas imponer a la libertad de mi mente». Virginia Woolf

\section{INTRODUCCIÓN}

Este artículo trata de recoger los aspectos clave de una investigación orientada a la construcción de un ensayo crítico y reflexivo, cuya finalidad es defender la inclusión de un enfoque de género en las iniciativas de educación y desarrollo para el avance en una sociedad más justa e igualitaria. Dentro de los principales objetivos de dicha investigación aparece en primer plano comprender las aportaciones que, desde la perspectiva de género, se proporcionan a la mejora educativa de las niñas desde su comunidad, así como plantear el empoderamiento en clave feminista como una estrategia educativa y de mejora de la calidad de vida de las personas.

El origen de nuestro ensayo de discusión teórica se ubica en proyectos de cooperación interuniversitaria en Mozambique, llevados a cabo por el equipo de investigación complutense al cual pertenecemos, y que han tenido como resultado la elaboración de un diagnóstico crítico (Cabello, Martínez y Sánchez, 2012; Cabello y Martínez, 2013) sobre la educación en dicho país. Centrándonos en las necesidades en materia de género detectadas, proponemos la elaboración de un enfoque de género que solvente desigualdades educativas y ayude a construir un nuevo enfoque teórico para la educación de las niñas.

El tratamiento que damos a los contenidos se enmarca dentro de un enfoque postcolonial y crítico en el estudio de las desigualdades sociales contemporáneas. Este enfoque cuestiona las lógicas basadas en jerarquías de poder global, racial, de género, epistémicas, pedagógicas... Y este cuestionamiento nos lleva a plantear enfoques alternativos de estudio, entre los cuales aparecen con especial relevancia dos temáticas: feminismo postcolonial y enfoque de género; y transculturalidad y africanismo. Encontrar las conexiones entre las mismas con una perspectiva de educación transformadora es un eje fundamental en nuestro trabajo.

La discusión de los resultados y conclusiones obtenidos nos permite definir cómo los términos relativos a perspectiva de género, desarrollo, empoderamiento y educación son constituyentes de una forma particular de saber, ser y hacer a la que no es ajena la educación. Construir un discurso teórico y práctico desde un enfoque de género nos lleva a estudiar lo que dicho enfoque tiene de particular, haciendo referencia a los intereses y necesidades de género. 


\section{Contextualización del estudio: transmisión del género a través de la educación, el desarrollo y la cultura}

Cabe iniciar el contenido de este artículo afirmando que el género existe, al menos en las relaciones entre las personas y las sociedades. Dicho concepto hace referencia a las relaciones de poder entre hombres y mujeres, causa de grandes desigualdades, sean en el ámbito individual o colectivo y consecuencia del aprendizaje, la cultura y la sociedad.

Esta idea se enmarca en las reflexiones de Lagarde (2012) que entiende el género como aquello que está presente en el mundo, en las sociedades, en los sujetos, en sus relaciones y en la política. Son importantes las aportaciones que la antropóloga americana Rubin (1986), citada por Oliva (2007) hace al referirse al «sistema sexo-género» considerando el género como «una divisoria impuesta socialmente a partir de relaciones de poder. Divisoria que asigna espacios, tareas, deseos, derechos, obligaciones y prestigio. Es decir, además de como categoría analítica, se considera un sistema de organización social» (p. 27).

Vieitez, Martín y Rodríguez (2012) ahondando en los estudios de género y desarrollo apoyan y analizan la peculiar relación entre género, cultura y sociedad. La cultura es entendida como el conjunto de diferentes formas de vida, significados y valores, siendo heterogénea, diversa y en continuo movimiento. La categoría de género, en este marco, se vincula con el orden cultural y con una determinada organización social en la que las mujeres están en una posición desigual con respecto a los hombres. Es decir, los valores culturales van a influir en la construcción de las percepciones de desarrollo y de género y van a condicionar la respuesta local y global a estos aspectos.

Tripp (2008) hace una dura crítica hacia aquellas sociedades que defienden prácticas culturales que perjudican a las mujeres en nombre de la preservación de su identidad religiosa, étnica o cultural, muchas veces buscando proteger ciertos intereses políticos, económicos... lo que ha llevado a la «culturalización» de los debates en torno al género y los derechos de las mujeres. Vieitez $(2013,2014 a)$, siguiendo a la autora anterior, reivindica que las prácticas culturales dañinas para las mujeres deben ser tratadas como un problema político más amplio, no limitando la intervención feminista a las reformas legales en exclusiva, sino dando valor, por ejemplo, a los trabajos sobre educación.

En relación con esta idea de cultura encontramos los intereses estratégicos de género, basados en la posición de género (desigual ubicación social, política, económica y cultural de las mujeres respecto de los hombres) en un 
contexto determinado. Tener en cuenta estos intereses estratégicos será un elemento definitorio de la inclusión del enfoque de género en la educación, como base para la trasformación de las estructuras sociales desiguales; ya que supone una concepción de la educación más allá de un enfoque de necesidades limitado a atender demandas puntuales (alfabetización, aumento de matriculaciones...), para pasar a un plano más complejo centrado en un enfoque de derechos. De esta manera se propicia un proceso de apoyo a la toma de conciencia y la adquisición de habilidades necesarias para una práctica transformadora. Según lo anterior, la acción educativa viene acompañada, además de por la toma de conciencia, por un cambio en las estructuras sociales y culturales desde las propias bases sociales a través de procesos participativos.

Nos proponemos seguidamente desarrollar esta última idea, definiendo las aportaciones que conlleva la inclusión de una perspectiva de género crítica en la educación y el desarrollo para la mejora educativa de las niñas.

\section{MARCO METODOLÓGICO}

El marco metodológico seguido responde a técnicas propias de la investigación social cualitativa, específicamente la denominada teoría fundamentada, al entender el mundo social como un proceso en continua construcción. Se ha seguido un método inductivo y comparativo en el procesamiento de la información, con un procedimiento circular de análisis y síntesis para el debate teórico a lo largo de todo el estudio; cuya finalidad ha sido deconstruir las teorías, saberes y enfoques dominantes para su reinterpretación y reconstrucción.

Los criterios seguidos para la selección y estudio de las fuentes revisadas se han basado: en los años de publicación (desde los '90 al 2014), en la temática apropiada a la finalidad del tema a discutir (género, desarrollo y educación), en el área geográfica predeterminada (África) y en los enfoques coherentes a nuestra propuesta (descolonización de saberes).

\section{PRESENTACIÓN DE RESULTADOS Y DISCUSIÓN TEÓRICA}

Entre las contribuciones al análisis de las desigualdades sociales, desde los enfoques de descolonización de los saberes, el feminismo postcolonial tiene un protagonismo cada vez mayor, aportando nuevos argumentos al cuestionamiento del orden dominante mundial. A continuación, sinteti- 
zamos las principales aportaciones de esta corriente de pensamiento a la comprensión de la desigualdad de género en la educación y el desarrollo.

\section{Descolonizando el feminismo para la construcción de un enfoque de género en la educación}

$\mathrm{Al}$ margen de las particularidades históricas, los feminismos comúnmente llamados occidentales han tenido una importante influencia en los análisis de género en todas las partes del mundo. Sobre todo, en materia de igualdad, de visibilización de la mujer, de emancipación o de denuncia de las desigualdades sexistas; sin embargo, al incorporar miradas descolonizadoras en estos análisis de género, se acusa a este feminismo de imponer un determinado modelo de ser mujer, y de entender las relaciones de género de una manera etnocéntrica, "sin dejar a África libertad de pensamiento con respecto a las relaciones entre hombres y mujeres» (Traoré, 2013, p. 132).

Estas corrientes de pensamiento feminista comienzan a «reivindicar identidades culturales propias a la vez que se enfrentan a las tradiciones patriarcales» (Suárez y Hernández, 2008, p. 15). Las autoras denuncian que los feminismos «occidentales se asuman como mundiales, ignorando los efectos del colonialismo y el imperialismo en la vida de las mujeres» (Suárez y Hernández, 2008, p. 15).

Cabe sintetizar las tesis del feminismo postcolonial en el pensamiento de Mohanty (2008) y en su apreciación de que «quizás, ya no se trate simplemente de los ojos de occidente» sino de cómo "occidente está dentro y reconfigura constantemente y globalmente los términos de raza y género» (Mohanty, 2008, p. 432). La misma autora sitúa a los feminismos postcoloniales en pro de alianzas híbridas, multiétnicas y transnacionales.

En este contexto de crítica, los feminismos africanos comparten con los feminismos postcoloniales (Gallardo, 2014) debates acerca de los modelos de ser mujer y ser hombre impuestos desde la ideología dominante del capitalismo patriarcal occidental, primero por la colonización y más adelante por la globalización, concluyendo que ambos modelos de organización social han dado lugar a ideas homogéneas de cultura, religión o sociedad en la mayoría de países del mundo.

Dicha corriente, según Grosfoguel (2013), se define por una perspectiva «interseccional ${ }^{2}$ » ante las diferentes formas de opresión y reproducción de los ejes de poder que forman parte de nuestra civilización. El autor señala que la lucha contra el modelo capitalista y patriarcal requiere de una organización antisexista, antirracista, antieurocéntrica, antiimperialista y antico- 
lonial. Siguiendo esta lógica, luchar contra el patriarcado y la globalización eurocéntrica requiere organizarse de manera «interseccional» evitando así que se vuelvan a reproducir las lógicas de dominación occidental. Ello justifica la pertinencia de descolonizar el sistema-mundo capitalista/patriarcal; moderno-colonial.

En este marco de reivindicaciones feministas, desde un punto de vista intercultural y africano, se reconoce la necesidad de descolonizar un feminismo europeo, etnocéntrico y universalista, para lograr una «lectura de los problemas de las mujeres desde sus propias realidades, es decir, sin abandonar su cultura y mundo social» (Kabunda, 2009, p. 222).

\section{Construcción y socialización del género desde el feminismo africano}

Al hablar de diferencias entre los feminismos occidentales y los africanos conviene no perder de vista los puntos comunes y de interseccionalidad $^{1}$, entre los que cabe recordar las semejanzas en torno a la defensa y garantía de los derechos humanos y de las mujeres, la visibilización de las mismas, o la toma de conciencia ante las desigualdades. Entre las diferencias, por tener una influencia primordial en el desarrollo educativo, destacaremos las aportaciones que se hacen desde los feminismos africanos sobre la construcción y socialización del género.

Tomando como referencia la idea de género como constructo cultural y atendiendo a las particularidades de cada realidad, la antropóloga nigeriana, Amadiume (1997) entiende que el género está formado por categorías flexibles, las cuales tienen como resultado diferentes formas de relación y de poder; el respeto a la diversidad de cada contexto es la base del pensamiento de la autora. Esta misma tesis es apoyada por otras autoras, como la feminista africana Oyèwumi (2010) quien critica el uso generalizado de «mujer» como categoría social, sin poder y definida en relación con al varón; y defiende la necesidad de teorizar sobre las diversas formas de desigualdad (raza, género, clase, edad o etnia) en la organización social, apoyándose en la afirmación de que «el género no puede abstraerse del contexto social y de otros sistemas de jerarquía» (Oyèwumi, 2010, p. 28).

Mobolanle (2011) señala que interpretar las realidades de las mujeres africanas según conceptos feministas occidentales puede llevar a distorsiones y falta de comprensión, por ello el feminismo africano aboga por repensar desde África las categorías de género y mujer, oponiéndose al pensamiento dominante. Jabardo (2012) y Vieitez (2013 y 2014a) defienden que no todos estamos globalizados de la misma manera (conexiones sur-sur, norte-norte, norte-sur), de ahí la preocupación por entender el género desde 
su diversidad con el fin de elaborar una epistemología alternativa y contrahegemónica.

Las conceptualizaciones basadas en el etnocentrismo cultural sobre género y desarrollo generan por parte de los feminismos africanos - apoyados por la crítica elaborada por los feminismos postcoloniales- la reivindicación de un desarrollo transformador, que tenga en cuenta las características particulares de cada contexto evitando, entre otros aspectos, las generalizaciones.

Estas especificidades en la construcción de conocimiento en general y de la idea de género en particular remiten a la forma de entender lo cultural y las pautas sociales de cada comunidad y adquieren especial relevancia ante el propósito de incluir un enfoque de género en la educación. A continuación presentaremos algunas aportaciones prácticas que dicho pensamiento genera en la acción educativa para el cambio social pro-equidad.

\section{NOTAS CONCLUSIVAS PARA UNA ACCIÓN EDUCATIVA EMANCIPADORA Y CON PERSPECTIVA DE GÉNERO}

$\mathrm{Al}$ observar la actualidad con una mirada que busca la equidad comprobamos que la desigualdad de género está muy presente en nuestras sociedades, tanto en la educación (socialización desigual de niños y niñas, desiguales orientaciones en la formación profesional, falta de perspectiva de género en la formación de formadores...), como en el empleo, la política o la economía. El enfoque de género sitúa la causa de esta desigualdad en una recolonización del patriarcado unida a la extensión de un modelo socioeconómico globalizado en la desigualdad. En consecuencia, las estrategias feministas para el desarrollo, además de plantear un enfoque crítico de análisis, incluyen una perspectiva de género transversal en la acción, siendo la actividad educativa un factor determinante.

Los significados que el feminismo postcolonial otorga a la perspectiva de género, están íntimamente relacionados con un enfoque de derechos y, por lo tanto, con un determinado paradigma social. Unir la perspectiva de género a un enfoque de derechos interdependientes, tal y como los entiende Tomasevski (2004), nos facilitará un análisis crítico y feminista sobre las relaciones desiguales entre mujeres y hombres, sobre las normas, las creencias y todo un mundo simbólico encargado de reproducir y perpetuar estas desigualdades en cada sociedad. Hemos identificado también, como aporte de esta perspectiva, que lo importante no es señalar la desigualdad de género como el problema en sí; sino atender a los intereses estratégicos de género y a una concepción de desarrollo transformadora, lo que establecerá el origen 
del problema en una «base cultural que sostenga las estructuras de poder de la comunidad, siendo la causa de las resistencias al cambio» (Rodríguez y Solange, 2009, p. 514).

Desde dichas aportaciones y en coherencia con la finalidad de este artículo, tratamos de evidenciar y defender los beneficios que la inclusión de esta perspectiva de género aporta a la educación (Martínez, 2016), atendiendo al propósito de generar los cambios necesarios en estructuras sociales y culturales que están impidiendo el desarrollo en equidad de las niñas y niños.

Centrar nuestro ámbito de acción en la intervención educativa y social supone hablar de cambios culturales, aunque lograr esta tarea sin caer en posturas etnocéntricas, relativistas o de interferencia cultural, acarrea una gran complejidad. Rodríguez y Solange (2009) teniendo en cuenta que toda cultura es dinámica, abierta y heterogénea, señalan que los valores culturales son reinterpretados en función de las necesidades de cada sociedad. Las autoras afirman que para que se produzca un cambio cultural y social proequidad es necesario un cuestionamiento cultural por parte de las propias mujeres y de la sociedad en su conjunto.

El enfoque feminista y de derechos —en el que se ubica este artículoconsidera la desigualdad de género como un conflicto cultural, de intereses económicos, políticos y, en definitiva, de poderes; y en consecuencia la defensa de los derechos individuales de las personas, hombres y mujeres, ha de ser compatible con el respeto por los derechos de los pueblos. Las incompatibilidades aparecerán con aquellas personas que sustentan el poder de manera desigual.

Tripp (2008) ubicándose en un feminismo postcolonial y como resultado de su investigación en Uganda, afirma que se apela a la tradición cultural como argumento contra ciertos derechos de las mujeres. Destaca la lucha contra la mutilación genital femenina en Uganda o Senegal, y refiere experiencias donde las mujeres se convirtieron en agentes de cambio de sus propias comunidades, adquiriendo la educación un papel fundamental.

Tomando este ejemplo como referencia, toma fuerza el hecho de que la lucha por la igualdad de género es política y debe ser abordada por las mismas personas que resulten discriminadas. Las sociedades, por lo tanto, no son homogéneas, ni culturalmente coherentes, al estar compuestas por personas dinámicas, cambiantes, diversas... Sostenemos que, en general, la cultura es una construcción social y, por tanto, que los cambios culturales requieren tiempo para que las dinámicas de género no permanezcan intactas. 
Para que dichas prácticas transformadoras se asienten en la vida cotidiana de la comunidad y los cambios en los mandatos de género sean estables, será necesaria una constancia en el tiempo y una acción social multidimensional, integral e interseccional.

Según un estudio de Crenshaw (2002-2004) avalado por AWID, repensar nuestras acciones desde una perspectiva interseccional tendrá como objetivo visibilizar las múltiples identidades que interactúan en los diferentes tipos de discriminación, considerando para ello los contextos históricos, sociales y políticos, junto con las experiencias individuales. Por ejemplo, no será cualitativamente igual la experiencia de una mujer negra en Ciudad del Cabo a la de una mujer blanca o indígena. Este análisis interseccional no tendrá como objetivo mostrar cómo un grupo está más victimizado que otro, sino descubrir las diferencias y similitudes significativas para poder superar las discriminaciones y establecer las condiciones necesarias para el cumplimiento de los derechos humanos en equidad.

Como paradigma teórico la interseccionalidad (Jabardo, 2012) permite, además de entender las situaciones de opresión desde la diversidad, construir acciones en favor de la igualdad partiendo de la transversalidad. Esta idea es apoyada por el feminismo africano al hacer hincapié en el aporte de estrategias alternativas para construir una cultura de los derechos humanos en igualdad, en todos los niveles — desde lo local a lo global — atendiendo a los contextos particulares; no tratándose tanto de lograr identificar cada forma de opresión, sino de conseguir que cada persona sea respetada.

Para sumar esta perspectiva a nuestra acción educativa tendremos que repensar desde otros puntos de vista los aspectos relacionados con la identidad, la igualdad, la justicia o el poder. Esto supondrá no limitar nuestra investigación y acción a categorías cerradas y aisladas desde un sector, sino incorporarnos a las aportaciones de otros sectores para lograr definir el acceso a los derechos y a las oportunidades, preguntándonos cómo realmente viven sus vidas las mujeres y hombres en cada comunidad desde la complejidad.

Por todo ello, la acción educativa (Martínez, 2016) con perspectiva de género en tanto que socializa, transforma, genera ciudadanías responsables y pone en actividad capacidades y habilidades tanto individuales como grupales, cobra una importancia fundamental en este proceso de transformación cultural hacia la equidad. Desde la perspectiva adoptada en este trabajo y con los aportes del enfoque de género y de derechos, la educación y la comunidad se convierten en una herramienta esencial para promover cambios culturales relativos a los saberes y las prácticas opresoras. 
Un aspecto a destacar respecto a la inclusión de una perspectiva de género en el desarrollo y la educación es la superación de las visiones simplistas de la realidad, que asumen la pobreza como un indicador comparativo entre países, el desarrollo como una carrera por ser el más rico, o la feminización de la pobreza como una característica intrínseca de las mujeres, solo por ser mujeres. No tener en cuenta esta perspectiva de género limitará los estudios de desarrollo a una comparativa entre indicadores unidimensionales, dedicados a clasificar a la población por sus carencias y no por sus oportunidades.

Hasta el momento, podemos concluir que repensar la educación y el desarrollo en clave feminista y de derechos facilitará: buscar, entender y definir las causas de las desigualdades de género, y no solo las consecuencias; interrelacionar las dimensiones intervinientes en el desarrollo en clave de género y descubrir formas y experiencias para romper con las dinámicas que sustentan estas prácticas desiguales; visibilizar y valorar las acciones realizadas por mujeres y hombres en pro de la igualdad; generar espacios para la transformación cultural; ubicar la educación como clave en estos procesos; $\mathrm{y}$, en definitiva, cooperar en el espacio en que actuamos como profesionales para abrir un camino hacia una justicia social equitativa.

\section{Empoderamiento, finalidad educativa}

En este repensar la educación con mirada de género aparece con cierta relevancia el concepto de empoderamiento. Siguiendo a Vieitez y Manzanera (2014b) y a pesar de ser un término asociado al desarrollo occidental, nos referimos a este cuando atendemos a las capacidades, individuales y globales, de pensar y actuar libremente, es decir, al proceso por el cual una mujer o grupo social adquiere poder al ser capaz de tomar decisiones, elegir y ser elegida, participar en lo social y político, educar y educarse, gozar de seguridad en el empleo, etc. Ha sido habitual asociar el término empoderamiento al ámbito económico en exclusiva; sin embargo en este artículo, siguiendo aportaciones del análisis de género, vamos a comprobar cómo el concepto va más allá al cuestionarnos si, como señala Smyth (2010), nuestras prácticas a favor del empoderamiento están generando espacios para la transformación de las conciencias de las personas y sus comunidades.

Siguiendo las reflexiones de las feministas africanas expuestas, el empoderamiento es entendido como la toma de voz propia, Oyèwumi (2010) piensa el concepto como el proceso que otorga a la mujer poder en los asuntos públicos, pero también en los privados. 
Observamos que el término empoderamiento, en sus orígenes, ha sido usado en diferentes contextos para referirse a la liberación y concienciación de las clases oprimidas. Siguiendo los argumentos de Gramsci, Foucault o Freire, podemos aplicar el término a cualquier grupo que se considere vulnerable socialmente, si entendemos el mismo como toma de poder ante la opresión y el poder como el acceso, uso y control de recursos tanto físicos como ideológicos, en una relación social. Freire (2012) utiliza este concepto para referirse a un mecanismo mediante el cual se desarrolla un cambio de mentalidad, siendo este proceso una condición necesaria para alcanzar una acción transformadora y de liberación de los oprimidos. El autor otorga a la educación el papel de concientización sobre las contradicciones y desigualdades del mundo y a la enseñanza la posibilidad para aprender y desarrollar las capacidades necesarias para ejercer el poder de manera igualitaria.

Aun considerando una gran variedad de definiciones y análisis sobre el concepto de empoderamiento, Kabeer (1998) afirma que nos empoderamos individual y colectivamente. La dimensión individual del empoderamiento engloba el trabajo personal de cada mujer o niña para concienciarse y reconocerse en sus capacidades y habilidades y en cómo usarlas para alcanzar su autonomía, más allá del acceso al empleo. En esta dimensión individual cobra una especial importancia el fomento de actuaciones educativas de formación y capacitación, que garanticen un desarrollo pleno de las capacidades de toma de conciencia personal y como ciudadanas. Por otro lado, la dimensión colectiva parte de la unión o creación de redes por parte de un grupo de mujeres en torno a objetivos comunes. Estos objetivos son los que refuerzan su proceso de empoderamiento. En esta última dimensión encontramos dos niveles de actuación: político — visibilización, transformación...- y económico - acceso a recursos y fondos, libertad...-(García, 2009).

Englobando estas definiciones, según ONU-mujeres (2011-2012) empoderarse implica, además de tener iguales capacidades (educación, salud), igual acceso y uso de recursos y poder utilizar esos derechos, capacidades, recursos... libres de toda violencia y coerción.

Encontrar proyectos que tengan como objetivo principal el empoderamiento de sociedades, de grupos minoritarios o de excluidos, es cada vez más habitual; pero alcanzar la culminación de los mismos con éxito, más allá de resultados parciales, se convierte en una tarea compleja y no siempre realizable. Incluir el empoderamiento como objetivo de las acciones sociales y educativas conllevará un trabajo multidimensional, coherente, simultáneo y participativo, es decir, un trabajo que integre las demandas, facilite espacios de reflexión y diálogo, trabaje desde el respeto... Rodríguez y Teijo (2009) completa esta idea con la recomendación de invertir en las capacidades de las mujeres y empoderarlas para que ejerzan sus opciones, no solo 
como un fin valioso en sí mismo, sino también como la manera más segura de contribuir al crecimiento económico y al desarrollo general.

En este sentido García (2010, p. 25) afirma que «la mejora de la posición de las mujeres, la toma de poder y conciencia, el ejercicio de sus derechos humanos, dignifica y mejora la sociedad, implica un crecimiento para todas y todos y forma parte de los principios de la democracia». A ello Lagarde (1996) añade que para potenciar la capacidad democratizadora del mundo es fundamental lograr el empoderamiento personal y colectivo de las mujeres.

Por lo tanto, el empoderamiento hace referencia no solo a un fin individual y social, sino también a un proceso complejo, que depende de relaciones cambiantes de poder y de género. En este artículo el empoderamiento es entendido como un fin y un medio educativo para la mejora de las condiciones de vida de mujeres y niñas, que requiere ser abordado desde un punto de vista multidimensional y transversal. Los procesos encaminados a conseguir que las personas y las comunidades adquieran empoderamiento tendrán que ser acompañados de una educación facilitadora, es decir, transformadora, emancipadora, crítica y de calidad.

\section{DISCUSIÓN FINAL: HACIA UNA EDUCACIÓN CRÍTICA Y EN EQUIDAD}

Este artículo se enmarca en trabajos de investigación africanos y africanistas que se están llevando a cabo en relación con la construcción de un conocimiento propio y crítico sobre África, más allá de las aportaciones que desde la cooperación y los saberes dominantes se hacen. Se trata de un paradigma alternativo que propone descolonizar el conocimiento en torno a la educación y, a la vez, trata de dar respuesta a una de las principales injusticias sociales de nuestra época: la desigualdad y en nuestro caso, la desigualdad de género. Nuestro trabajo comparte con estos enfoques alternativos la convicción de que el cambio social es posible, que la educación es un factor imprescindible en el mismo, y que no puede hacerse al margen del respeto a la diversidad, motivaciones, intereses, iniciativas y demandas de las personas interesadas.

Robert (2007) reivindica, desde su análisis alternativo de las relaciones sociales entre África y Occidente, una forma diferente de participar en el mundo al margen del capitalismo dominante. Para ello, la autora plantea como necesario generar procesos de integración y de diálogo respetuosos, basados en el conocimiento mutuo, abiertos a la dignidad humana y dando voz a los y las excluidas. Estos enfoques alternativos — altermundismo, bien 
común, africanismo...- señalan nuevas vías para un humanismo enriquecedor, crítico y transformador. Dentro de esta perspectiva la apuesta por una educación de y para todos y todas adquiere un papel fundamental en la toma de conciencia crítica en favor de la justicia social.

En este sentido Sousa (2010) se refiere a la necesidad de reinterpretar la concepción de los derechos humanos desde la interculturalidad. Para ello será necesario reconocer la diversidad de saberes, interpretaciones, formas de hacer y de pensar existentes en cada sociedad. Entre otros aspectos destaca el derecho a la diversidad del conocimiento, a la propiedad orientada a la solidaridad, a la autodeterminación democrática y a la participación en la creación de derechos. El autor sienta las bases para la construcción intercultural de una política emancipadora de derechos humanos, la cual debe basarse en la lucha por la democratización, la desmercantilización y la descolonización.

\section{Propuestas para una práctica educativa crítica, postcolonial y feminista}

Bajo estos principios, la inclusión de una perspectiva que integra género y derechos aporta nuevas formas de hacer y pensar las acciones de desarrollo, educativas y sociales, generando procesos de enseñanza y aprendizaje en clave feminista. Estas transformaciones serán visibles en los significados de la acción educativa sobre cada práctica, al procurar nuevas formar de saber, ser y hacer, en los complejos procesos que forman parte de la tarea educadora desde su interdimensionalidad: desigualdades de género en el curriculum, socialización, violencia estructural o simbólica, formación de profesionales, construcción de materiales, relaciones institucionales, organización de espacios, tiempos, contenidos, etc.

Los estudios y análisis realizados justifican la reivindicación de una educación para la equidad, como espacio generador de alianzas y transformaciones estratégicas en pro de un desarrollo alternativo, que vaya más allá de la visión mercantilizada de la educación y favorezca alcanzar los objetivos de la educación para todos y todas en igualdad.

Señalamos que para alcanzar estos objetivos es necesario reivindicar y repensar la relación entre desarrollo, ciudadanía y educación, considerando esta última como oportunidad, al mismo tiempo que como condicionante y posibilidad de reconstrucción de nuevas ciudadanías. Defendemos la escuela y los espacios educativos como esferas públicas democráticas, lo que lleva a la participación consciente en la toma de decisiones, con compromiso, diálogo abierto y horizontal; a la educación permanente para todos y 
todas, ofreciendo oportunidades para aprender a hacer, sentir y ser ciudadanos, ampliando los espacios y tiempos de educación; y a posibilitar a todos $\mathrm{y}$ todas el acceso al ejercicio pleno de los derechos universales.

Según los anteriores análisis, una intervención educativa que favorezca procesos de enseñanza y aprendizaje igualitarios debe orientarse hacia un desarrollo local, a la vez que global, como forma de promoción humana que contemple la cultura de cada contexto concreto, sus posibilidades y limitaciones, hacia el empoderamiento. De esta forma se favorecerá el crecimiento personal, la expansión de las capacidades individuales y grupales, la ampliación de la gama de alternativas, el logro de libertades, la toma de conciencia y la compresión de los problemas y de sus causas..., promoviendo de esta manera, la organización popular y el fortalecimiento del tejido social para la acción, sin incurrir en los riesgos del neocolonialismo occidental que denuncian los feminismos descritos.

Las prácticas educativas desarrolladas bajo este enfoque se caracterizarán por los siguientes rasgos definitorios: a) horizontalidad en las intervenciones, b) visibilización de las imposiciones del patriarcado, c) generación de capacidad autocrítica, de toma de conciencia individual y colectiva, a partir de la potenciación de las habilidades propias, d) orientación prioritaria a facilitar procesos de empoderamiento individuales y colectivos, e) divulgación y explicación de la historia, la cultura, los saberes... desde un punto de vista feminista e intercultural, descubriendo el valor de conocimientos ocultos o poco apreciados y no solo de los dominantes y globalizadores, f) énfasis en el fomento de redes de mujeres en pro de un modelo social basado en el bien común, en el apoyo mutuo entre personas, en generar redes solidarias (por ejemplo, redes de préstamo de dinero entre mujeres: tontines en Senegal o Xitique en Mozambique), g) sinergias con movimientos que promuevan la formación, divulgación y acción social con perspectiva de género.

Como se ha visto, partir de la inclusión del enfoque de género en el desarrollo exigirá una determinada forma de pensar la intervención socioeducativa y, por lo tanto, una forma particular de entender la educación: equitativa, crítica, participativa, transformadora, feminista... Los discursos elaborados con este enfoque pretenden «dotar a las mujeres (y niñas) de un lugar desde el cual hablar y del poder para hacerlo, identificando la multiplicidad de voces auténticas de cada uno y cada una» (Belausteguigoitia y Mingo, 1999, p. 67-73). En esta misma línea, uniendo la pedagogía crítica con la educación feminista, encontramos la necesidad urgente de repensar y reformar las prácticas educativas hacia prácticas que empoderen. Por ello, dentro de las propuestas de esta pedagogía, se incluye proveer a los profesionales de la educación y estudiantes de las habilidades que necesitarán 
para ubicarse en la historia, encontrar sus propias voces y establecer las convicciones necesarias para practicar el valor cívico y las relaciones democráticas en equidad.

En este sentido, la educación se convierte en una herramienta imprescindible para «cooperar en devolver a las personas capacidades de discurso y de acción, que les permitan apropiarse de sus destinos y actuar como ciudadanos y ciudadanas gobernantes y no como súbditos» (Cabello y García, 2006, p. 37).

Consideramos necesario insistir en que superar las desigualdades de género solo será posible si contamos con una educación entendida como un proceso de enseñanza y aprendizaje transformador y crítico que promueve prácticas de libertad y de poder igualitario, convirtiendo el acto de educar en una práctica moral de equidad. Práctica que impedirá que la educación privilegie a una persona sobre otra, cualquiera que sea su sexo, porque cuando esto ocurre, como afirma Connell (1997) se está dando a la primera una educación corrupta y, además, se está degradando la educación de todos y todas.

Finalmente, pensando en una acción educativa comprometida con los presupuestos anteriores, reivindicamos la construcción de una pedagogía feminista como garante del desarrollo social y de las capacidades de hombres y mujeres en equidad, para alcanzar resultados consistentes y potenciadores de justicia social para otro desarrollo posible.

Cabe cerrar estas reflexiones con unas palabras de la ex-ministra de educación de Mozambique, la señora Graça Michele, defendiendo que:

"...se necesita del compromiso de todos y todas para que estos cambios no sean aislados, es por ello que hay que situar el empoderamiento dentro del ámbito de los derechos humanos. (...) Un poder para la inclusión, para la justicia, para el desarrollo, para la paz (...) Ello nos lleva a: la educación, la formación y la cultura» (Michele, 2006).

\section{NOTAS}

1 La interseccionalidad es conocida como una teoría sociológica que analiza cómo diferentes categorías de discriminación interactúan en múltiples y simultáneos niveles. Surge a finales de los años '60 a la par del movimiento feminista multirracial. Estas feministas trataron de comprender las diferentes formas de interacción entre la raza, el sexo y la clase. (Hill Collins, P. (2000). Intersecting Oppresions [en línea]. Recuperado de: http://www.uk.sagepub. com/upm-data/13299_Chapter_16_Web_Byte_Patricia_Hill_Collins.pdf). 


\section{REFERENCIAS BIBLIOGRÁFICAS}

Amadiume, I. (1997). Reinv1enting Africa: Matriarchy, Religion and Culture. USA: Zed Books.

Belausteguigoitia, M. y Mingo, A. (1999). Géneros Prófugos. Feminismo y Educación. México: Paidós.

Cabello, M.J. y García, E. (2006). Fracaso escolar y fracaso vital. Hacia una reconceptualización del fracaso escolar que transforme su tratamiento. Revista Interamericana de Educación de Adultos, 1, 75-100.

Cabello, M.J., Martínez, I. y Sánchez, J.M. (2012). Formación continua del profesorado a través de una red de oficinas pedagógicas en Mozambique. Una propuesta desde los vínculos educación-desarrollo. Revista Educaçao y cultura contemporánea, 9, 4-24.

Cabello, M.J. y Martínez, I (2013). Inclusión de una perspectiva de género para la mejora de la educación básica en Mozambique. Revista Polifonías, 3, 79-96.

Connell, R. (1997). Escuela y Justicia Social. Madrid: Morata.

Crenshaw, K. (2002-2004). Interseccionalidad: Una herramienta para la justicia de género y la justicia económica. Derechos de las mujeres y cambio económico. Toronto: Association for Women's Rights in Development.

Freire, P. (2012). Pedagogía de la indignación. Buenos Aires: Editorial Siglo XXI.

Gallardo, F. (2014). Feminismos desde Abya Yala. Mexico: Corte y Confección.

García, C. (coord.) (2009). Encuentro de mujeres jóvenes África-España: empoderamiento y nuevos retos. Madrid: Fundación Mujeres.
García, B. (2010). Cooperar en femenino: políticas y prácticas de cooperación con perspectiva de género. Valencia: Tirant lo Blanch.

Grosfoguel, R. (2013). ¿Cómo luchar decolonialmente? Periódico Diagonal, 7. Recuperado de: http://goo.gl/Lses9o

Jabardo, M. (2012). Feminismos negros. Una antología. Madrid: Traficantes de Sueños.

Kabeer, N. (1998). Realidades Trastocadas. Las jerarquías de género en el pensamiento del desarrollo. México: Paidós.

Kabunda, M. (2009). Las mujeres en África: apuntes sobre los avances en sus derechos, logros y vulnerabilidades. En E. Molina y N. San Miguel, (eds.), Buenas Prácticas en Derechos Humanos de las Mujeres (pp. 217-237). Madrid: Oficina de Acción Solidaria y Cooperación, Universidad Autónoma de Madrid.

Lagarde, M. (1996). Género y Feminismo. Desarrollo Humano y Democracia. Madrid: Ed. Horas y Horas.

Lagarde, M. (2012). El feminismo en mi vida. Hitos, claves y utopía. México: Instituto de las Mujeres de Ciudad de México.

Martínez, I. (2016). Construcción de una pedagogía feminista para una ciudadanía transformadora y contra-hegemónica. Revista Foro de Educación, 20, 129-151.

Michele, G. (2006). Encuentro de Mujeres por un Mundo Mejor. [Conferencia. Archivo de video]. Recuperado de http://goo.gl/R9JLrC

Mobolanle, S. (2011). Feminismo: la búsqueda de una variante africana. Revista Africanenado: de actualidad y experiencias, 7, 17-27. 
Mohanty, C. (2008). Bajo los ojos de occidente: academia feminista y discursos coloniales. En L. Suarez, y A. Hernández, (Eds.), Descolonizando el feminismo: teorías y prácticas desde los márgenes (pp. 117-164). Madrid: Cátedra.

Oliva, A. (2007). Debates sobre el género. En C. Amorós, Teoría feminista: de la ilustración a la globalización (pp. 1557). Madrid: Minerva, Volumen 3.

ONU-Mujeres. (2011-2012). El progreso de las mujeres en el mundo. En busca de la justicia. New York: ONU-Mujeres.

Oyèwumi, O. (2010). Conceptualizando el género: Los fundamentos eurocéntricos de los conceptos feministas y el reto de la epistemología africana. $R e$ vista Africaneando: Revista de actualidad y experiencias, 4, 25-35.

Robert, C. (2007). África en Auxilio de occidente: saber hacer, saber vivir. Barcelona: Icaria Editorial.

Rodríguez, E. y Solange, M. (2009). Hacia el cambio cultural pro-equidad en el contexto de la cooperación al desarrollo en Mozambique. En E. Molina, y N. San Miguel, (eds.), Buenas Prácticas en Derechos Humanos de las Mujeres (pp. 507-525). Madrid: Oficina de Acción Solidaria y Cooperación, Universidad Autónoma de Madrid.

Rodríguez, I. y Teijo, C. (eds.) (2009). Ayuda al desarrollo, piezas para un puzzle. Madrid: La Catarata.

Sousa, B. (2010). Descolonizar el saber, reinventar el poder. Uruguay: Trilce

Smyth, I. (2010). Talking of gender: words and meaning in development organization. En A. Cornwall y D. Eade, (Eds.), Deconstructing Development Discourse. Buzzwords an Fuzzwords (pp.143-153). England: Oxfam.
Suarez, L. y Hernández, A. (eds.) (2008). Descolonizando el feminismo: teorías y prácticas desde los márgenes. Madrid: Cátedra.

Traoré, A. (2013). Estamos desbordados de potencialidades y me rebelo contra la naturaleza del sistema y su capacidad para destruir la esperanza en África. En A. Mama, M. Ogundipe, F. Alloo, F. Meer, A. Imam, Y. Jusu, Y. Mukagasana, et al., Africana: Aportaciones para la descolonización del feminismo. Selección de entrevistas (pp. 131-142). Barcelona: Oozebap.

Tripp, A. (2008). La política de los derechos de las mujeres y la diversidad cultural en Uganda. En L. Suarez, y A. Hernández, (Eds.), Descolonizando el feminismo: teorías y prácticas desde los márgenes (pp. 285-330). Madrid: Cátedra.

Tomasevski, K. (2004). El asalto a la educación. España: Intermon Oxfam.

Vieitez, S., Marín, I. y Rodríguez, J. (eds.) (2012). Percepciones del desarrollo dentro y fuera del continente africano. Granada: Universidad de Granada.

Vieitez, S. (2013). Movimientos africanos de mujeres y desarrollo. En A. Santamaría y J. Burgos, (coords.), Regreso al futuro: cultura y desarrollo en África (pp. 179-197). Madrid: La catarata.

Vieitez, S. (2014a). El África de las mujeres. Género y movimientos de mujeres. Madrid: Nociones comunes. Recuperado de: http://goo.gl/yTHF92

Vieitez, S. y Manzanera, R. (2014b). Género en el desarrollo y empoderamiento. En J. Ortega y C. Lubian, (coords.), Manual de educación para el desarrollo: orientaciones didácticas para el aula (pp. 53-68). España: Universidad de Jaén. 


\section{PERFIL ACADÉMICO Y PROFESIONAL DEL AUTOR/ES}

María Josefa Cabello Martínez. Profesora Emérita, Dpto. Didáctica y Organización Escolar, Facultad de Educación, Universidad Complutense. Líneas investigación: didáctica, formación del profesorado, educación de personas adultas, desarrollo comunitario, cooperación... Con este perfil ha sido: Investigadora Principal de Proyectos Competitivos de ámbito europeo, nacional e internacional. Directora y Codirectora de Tesis Doctorales y DEAs. Autora de libros y artículos publicados en revistas nacionales e internacionales. Ponente en congresos y actividades de ámbito internacional y nacional.

Irene Martínez Martín. Profesora asociada en Universidad Castilla la Mancha. Acreditada por Aneca como Ayudante Doctora. Ha sido becaria predoctoral FPU de investigación, Dpto Didáctica y Organización Escolar, Facultad de Educación, Universidad Complutense. Líneas de investigación principales: didáctica, educación permanente y formación del profesorado; feminismos en educación y desarrollo; educación y cooperación interuniversitaria para el desarrollo, específicamente, educación básica de las niñas en Mozambique; educación social. Con este perfil ha sido autora de artículos y ponente en actividades y congresos de ámbito nacional e internacional.

Dirección del autor/es: María Josefa Cabello Martínez

Calle Rector Royo Villanova, s/n

28040 Madrid

Email: josefac@edu.ucm.es

Irene Martínez Martín

Calle Madres de la Plaza de Mayo 2, portal 5. 5. ${ }^{\circ}$ B 28523 Rivas-Madrid

Email: irene.martinez@uclm.es

Fecha Recepción del Artículo: 06. Noviembre. 2013

Fecha modificación Artículo: 07. Abril. 2014

Fecha Aceptación del Artículo: 06. Mayo. 2014

Fecha Revisión para Publicación: 06. Julio. 2016 
